\title{
Appropriation Art: In Zeiten von „Metall auf Metall“ und des Internets
}

\author{
Christian Czychowski", Niclas Düstersiek ${ }^{* * *}$
}

\section{Einleitung}

Gute Künstler kopieren, große Künstler stehlen. ${ }^{1}$ Was dieses Zitat mit Witz anspricht, steht im Mittelpunkt der Appropriation Art: Appropriation Art, also die bewusste und mit Überlegung vorgenommene Übernahme fremder Werke in eigene Werke, gibt es im Urheberrecht schon sehr lange. Durch die zunehmende Verbreitung von Fotokunst hat sie vor etlicher Zeit eine neue Bedeutung bekommen.

Konnte man mit dem Phänomen in Deutschland vor dem Hintergrund der Germania 3-Rechtsprechung ${ }^{2}$ noch relativ gut umgehen, gerät die Diskussion durch die Metall auf Metall-Rechtsprechung ${ }^{3}$ des EuGH - genauso wie das frische BGH-Urteil Metall auf Metall $I V^{4}$ - auch rechtlich in ein neues Fahrwasser. Dies vor dem Hintergrund, dass Appropriation Art in der Kunst zurzeit gerade auch aufgrund von digitalen Techniken im Internet eine Renaissance erlebt. Der Beitrag soll die Anwendbarkeit und Praxistauglichkeit der neuen EuGH- und BGH-Rechtsprechung am Beispiel von Appropriation Art überprüfen und möglicherweise Lösungen unterbreiten, wie damit in Zukunft in rechtlicher Hinsicht umgegangen werden kann.

* Prof. Dr. RA, Christian Czychowski ist Fachanwalt für Informationstechnologierecht sowie für Urheber- und Medienrecht, und Honorarprofessor an der Universität Potsdam. Er arbeitet neben IT-, Urheber- und Medienrecht auch zu Softwarerecht und Technologievertragsrecht.

** Niclas Düstersiek ist Rechtsreferendar und wissenschaftlicher Mitarbeiter bei Nordemann Czychowski \& Partner Rechtsanwältinnen und Rechtsanwälte mbB mit Schwerpunkten im Urheberrecht, Internetrecht und in Open Source Software.

1 Teilweise Pablo Picasso zugeschrieben, genaue Herkunft unklar, Good Artists Copy; Great Artists Steal, https:/quoteinvestigator.com/2013/03/06/artists-steal/ (zuletzt abgerufen am 17.07.2020).

2 BVerfG, Beschl. v. 29.06.2000 - 1 BVR 825/98 - Germania 3.

3 EuGH, Urt. v. 29.07.2019 - C-476/17, NJW 2019, 2913 Rn. 39 - Metall auf Metall.

4 BGH, Urt. v. 30.04.2020 - I ZR 115/16 - Metall auf Metall IV. 


\section{Was ist Appropriation Art?}

Nach einem engen Verständnis handelt es sich um Appropriation Art, wenn Künstler bewusst und mit strategischer Überlegung Teile oder ganze Werke anderer Künstler kopieren, wobei der Akt des Kopierens und das Resultat selbst als Kunst verstanden werden sollten. Ein weiteres Verständnis hält jede Kunst für Appropriation Art, die sich mit vorgefundenem ästhetischen Material beschäftigt und dabei entweder nur kopiert oder in der Kopie Manipulationen vornimmt. ${ }^{5}$ Wichtig ist: Appropriation Art setzt nicht Betrug oder Täuschung voraus. ${ }^{6}$ Die Künstler orientieren sich in der Regel vielmehr offen an anderen Werken. Neben der möglichst identischen Übernahme fremder Werke in eigene Werke ist auch die teilweise Übernahme von juristischem Interesse, weswegen wir von einem weiten Verständnis ausgehen.

Appropriation Art als Prozess transzendiert Kunstrichtungen: In der Musik gibt es das „Musical Borrowing“" wie Sound Sampling und Mashups $^{8}$. Aber schon Beethoven bediente sich in seiner Eroica bei Motiven von Mozart. Beispiele dieser Art gibt es zu Hauf. Ein ähnlicher Begriff, dessen Spektrum sich mit Appropriation Art überschneidet, ist referenzielles Schaffen, wozu Kempfert neben Ausstellungen und Appropriation Art auch Samplings, Mashups, Remixes, Collagen, Parodien, Memes oder Fanfiction in digitalen Räumen zählt. ${ }^{9}$ Der Begriff Appropriation Art beschreibt damit den Prozess der Herstellung der Kunstwerke. Er verhängt, so wie wir ihn gebrauchen, kein Werturteil.

Appropriation Art ist in Zeiten des Internets und der zunehmenden Digitalisierung auch der Kunst ein Massenphänomen. Es wird zu klären sein,

5 Vgl. Zuschlag, „Die Kopie ist das Original“, in: Mensger, Déjà-vu?, 126, 127, 134; Tate, Art Term: Appropriation, https://www.tate.org.uk/art/art-terms/a/appropriati on (zuletzt abgerufen am 17.07.2020); MoMa Learning, Appropriation, https:/www .moma.org/learn/moma_learning/themes/pop-art/appropriation/ (zuletzt abgerufen am 17.07.2020); https://de.wikipedia.org/wiki/Appropriation_Art (zuletzt abgerufen am 17.07.2020); Appropriation Art ausführlich begrifflich eingrenzend: Huttenlauch, Appropriation Art, $22 \mathrm{ff}$.

6 Huttenlauch, Appropriation Art, 28.

7 Burkholder The Journal of Musicology 2018, 223; Burkholder, Musical Borrowing and Reworking. An Annotated Bibliography, http://www.chmtl.indiana.edu/borro wing/ (zuletzt abgerufen am 17.07.2020).

8 Dazu Döhl UFITA 2019, 19, $20 \mathrm{ff}$.

9 Kempfert UFITA 2019, 123, 124; Saiz-Garcia UFITA 2019, 107, zu referenziellen Schaffenspraktiken nach spanischem Recht. 
ob die urheberrechtlichen Lösungswerkzeuge einem solchen Massenphänomen gerecht werden.

\section{Appropriation Art im Wandel der Zeit}

In Los Angeles wird jährlich das Film-„Festival of (In)Appropriation“ gefeiert - technisch reichen die Filme vom YouTube Supercut über analoge Eingriffe bis hin zu code-basierten digitalen Änderungen. ${ }^{10}$ Prägnant beschreibt Döhl den technischen Wandel im Musikbereich: „Der heranwachsende prototypische Komponist unserer Zeit sitzt heute nicht mehr an einem Klavier oder einer Gitarre, sondern vor einem Laptop. "11 Zum Streaming und Kauf stehen alleine in Deutschland 2,4 Millionen Musikalben zur Verfügung. ${ }^{12}$ Dateien können am Computer erstellt, kopiert und bearbeitet werden. Im Musikbereich steht beispielsweise fähige OpenSource-Produktionssoftware in Gestalt von Rosegarden, Ardour und Audacity bereit. ${ }^{13}$ Unabhängig von der rechtlichen Bewertung wird also deutlich: Appropriation wie z.B. Sound Sampling im Musikbereich ist durch die Digitalisierung in vielen Feldern deutlich einfacher geworden und weist eine niedrigere Zugangsschwelle auf. ${ }^{14}$

\section{Rechtliche Grundlagen und Bewertung}

Zwei Sekunden reichen aus. Oder ein Foto, ein Gemälde, ein übernommenes Motiv. Wer Appropriation Art betreibt, kann als Plagiateur dastehen oder sich - so wie Moses Pelham - jahrelangen Rechtsstreitigkeiten ausgesetzt sehen. Doch auch angesehene Künstler wie Andy Warhol praktizieren Appropriation Art. Wo liegen die Grenzen? Was ist gesellschaftlich akzeptiert und ist es dann auch rechtlich erlaubt? In welchem Rahmen kön-

10 Stromberg, A Film Festival Illuminates the Experimental Edge of Appropriation Cinema, https://hyperallergic.com/531520/festival-of-inappropriation-los-angeles-f ilmforum/ (zuletzt abgerufen am 17.07.2020).

11 Döhl UFITA 2019, 19, 20.

12 Bundesverband Musikindustrie, Musikindustrie in Zahlen 2018, S. 18, http://miz.or g/downloads/dokumente/950/BVMI-2018-jahrbuch-musikindustrie-in-zahlen.pdf (zuletzt abgerufen am 17.07.2020).

13 Dazu Döhl UFITA 2019, 19, 20.

14 So zum Sound Sampling Döhl UFITA 2019, 19, 21: „Sound Sampling ist zu kompositorischer Alltagskultur mit niedriger Zugangsschwelle geworden.” 
nen sich Künstler bewegen, deren Selbstverständnis es ist, altgediente Vorstellungen zu hinterfragen? Mit diesen Grenzen, sowohl juristischen als auch denen bekannter Kunst und gesellschaftlicher Wahrnehmung, spielt Appropriation Art.

Das rechtliche Fundament für Appropriation Art bilden das europäische und das deutsche Urheberrecht. Einerseits kann die Künstler:in durch Appropriation Art ein eigenes urheberrechtlich geschütztes Werk schaffen. Sie kann dabei aber auch Urheberrechte Dritter verletzen. Andere Rechte, die durch Appropriation Art unter Umständen betroffen sein können, zum Beispiel Marken- oder Persönlichkeitsrechte Dritter, nimmt der vorliegende Beitrag nicht in den Fokus.

\subsection{Die urheberrechtliche Prüfung im prototypischen Fall von Appropriation Art}

Die urheberrechtliche Prüfung im prototypischen Fall von Appropriation Art sieht wie folgt aus: Die genutzte Vorlage ist urheberrechtlich geschützt. Sie hat entweder Werkcharakter oder ist durch ein Leistungsschutzrecht geschützt wie die Tonaufnahmen im Fall Metall auf Metall durch $\mathbb{8} 85$ I 1 UrhG. Durch die Herstellung der Appropriation Art wird sodann in das jeweils betroffene Recht eingegriffen. Insbesondere können eine unerlaubte Bearbeitung, aber auch reine Vervielfältigungen vorliegen.

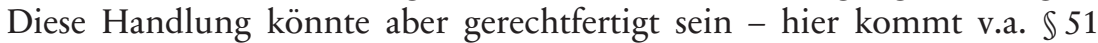
UrhG, das Zitatrecht, oder andere Schranken in Betracht sowie - ohne an dieser Stelle die dogmatische Einordnung des $\$ 24$ UrhG zu diskutieren eine erlaubte Nutzung i.S.d. $\$ 24$ I UrhG. Auch das Eingreifen weiterer Schranken ist denkbar.

\subsection{Die Germania 3-Rechtsprechung des Bundesverfassungsgerichts}

Im Fall Germania 3 ging es um das Theaterstück „GERMANIA 3 GESPENSTER AM TOTEN MANN“ von Heiner Müller. In einer Szene des Theaterstücks wurden, typisch für Appropriation Art, ohne Genehmigung Textpassagen aus Bühnenwerken Bertolt Brechts wiedergegeben, namentlich aus dem „Leben des Galilei“ und „Coriolan“. ${ }^{15}$ Diese ungefähr zwei Textseiten wurden in der Buchausgabe kursiv gekennzeichnet, während

15 Zu diesen „Zwischentexten“ anschaulich Metzger ZUM 2000, 924 f. m.w.N. 
der Anhang in allgemeiner Form auf die Quellen verwies. ${ }^{16}$ Das OLG München hatte ursprünglich eine Urheberrechtsverletzung angenommen. ${ }^{17}$

Im Zentrum des Beschlusses steht die praktische Konkordanz zwischen der Kunstfreiheit und dem das Urheberrecht umfassenden Schutz des Eigentums, Art. 5 III 1 GG bzw. Art. 14 I GG. Das Bundesverfassungsgericht hob die Entscheidung des OLG München auf und betonte die Reichweite der Zitatfreiheit bei Appropriation Art. Das OLG München setze sich mit dem künstlerischen Anliegen nicht hinreichend auseinander und lasse die durch Art. 5 III 1 GG gebotene kunstspezifische Betrachtung des von Müller geschaffenen Werkes vermissen. ${ }^{18}$ Dabei hob das Bundesverfassungsgericht insbesondere hervor, dass die Zitatfreiheit bei künstlerischen Sprachwerken einen weiteren Anwendungsbereich einnehme als bei nichtkünstlerischen. ${ }^{19}$ Ihr komme keine bloße Belegfunktion zu. ${ }^{20}$ In der notwendigen Gesamtbetrachtung erwähnte das Gericht die künstlerische und kritische Auseinandersetzung, das Interesse am Schaffensprozess im Dialog zu vorhandenen Werken, den eher geringen Eingriff in die Urheberrechte ohne Gefahr wirtschaftlicher Nachteile sowie den Charakter als Gestaltungsmittel. ${ }^{21}$ Beachtlich ist, dass die Zulässigkeit der Verwendung des fremden Textes im Rahmen eines Kunstwerkes nicht davon abhänge, „ob der Künstler sich damit ,auseinander setzt ${ }^{6}$, maßgeblich ist vielmehr allein, ob es sich funktional in die künstlerische Gestaltung und Intention seines Werks einfügt und damit als integraler Bestandteil einer eigenständigen

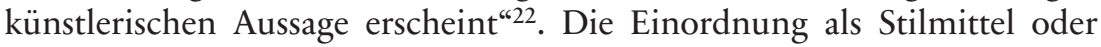
formale Kategorien wie „Kollagetechnik“ oder „Technik der zitierenden Kombination“ würden nichts über die künstlerische Bedeutung des Zitats aussagen. ${ }^{23}$

Zusammenfassend ist festzuhalten: Das Bundesverfassungsgericht verlagerte die Abwägung der Grundrechtspositionen im Germania 3-Beschluss auf die Schrankenebene und flexibilisierte die Zitatfreiheit für künstleri-

16 Zum Sachverhalt s. BVerfG, Beschl. v. 29.06.2000 - 1 BVR 825/98, Rn. 2 - Germania 3.

17 OLG München, Urt. v. 26.03.1998 - 29 U 5758/97, juris Rn. 36 f.

18 BVerfG, Beschl. v. 29.06.2000 - 1 BVR 825/98, Rn. 21, 25 - Germania 3.

19 BVerfG, Beschl. v. 29.06.2000 - 1 BVR 825/98, Rn. 22 - Germania 3.

20 BVerfG, Beschl. v. 29.06.2000 - 1 BVR 825/98, Rn. 22 - Germania 3; ausführlich zu Zitatzweck und -recht Metzger ZUM 2000, 924, $926 \mathrm{ff}$.

21 Vgl. BVerfG, Beschl. v. 29.06.2000 - 1 BVR 825/98, Rn. 22 ff. - Germania 3.

22 BVerfG, Beschl. v. 29.06.2000 - 1 BVR 825/98, Rn. 27 - Germania 3.

23 BVerfG, Beschl. v. 29.06.2000 - 1 BVR 825/98, Rn. 27 - Germania 3. 
sche Werke. Stehe ein geringfügiger Eingriff in die Urheberrechte ohne die Gefahr merklicher wirtschaftlicher Nachteile der künstlerischen Entfaltungsfreiheit gegenüber, so hätten die Verwertungsinteressen der Urheberrechtsinhaber im Vergleich zu den Nutzungsinteressen für eine künstlerische Auseinandersetzung zurückzutreten. ${ }^{24}$

\subsection{EuGH Metall auf Metall, BGH Metall auf Metall IV und die Auswirkungen auf das (deutsche) Urheberrecht und Appropriation Art}

Die Entscheidungen des EuGH in Metall auf Metall und aktuell des BGH in Metall auf Metall IV im Bereich des Sound Sampling sind nicht nur öffentlichkeitswirksam und gesellschaftlich kontrovers, sondern stellen für das Sound Sampling und Appropriation allgemein eine wichtige Weichenstellung dar. Wie diese aussieht und wie weit diese reicht, behandeln wir im folgenden Abschnitt, und zwar beispielhaft an den wichtigsten betroffenen Verwertungsrechten.

\subsubsection{Vervielfältigung}

Der EuGH stellt in seiner Entscheidung Metall auf Metall klar, dass die Übernahme von Audiofragmenten ${ }^{25}$ einen Eingriff in das ausschließliche Recht des Tonträgerherstellers nach Art. 2 lit. c RL 2001/29 darstelle, es sei denn, dass das Fragment in geänderter und beim Hören nicht wiedererkennbarer Form eingefügt werde. ${ }^{26}$ Nur dann keinen Eingriff anzunehmen, wenn das Fragment nicht wiedererkennbar ist, ist eine vergleichsweise strenge Grenzziehung. ${ }^{27}$

24 BVerfG, Beschl. v. 29.06.2000 - 1 BVR 825/98, Rn. 24 - Germania 3; krit. Schack FS Nordemann, 107, 109, der eine unmittelbare Berufung auf Art. 5 III 1 GG sieht und als dogmatisch verfehlt erachtet.

25 Kritisch gegenüber der weitgehend verwendeten Bezeichnung von Samples als Fragment durch den EuGH, da die Samplingkultur weit über die Verwendung kleinster Tonsequenzen hinausgeht: Döhl UFITA 2019, 19, 25.

26 EuGH, Urt. v. 29.07.2019 - C-476/17, NJW 2019, 2913 Rn. 39 - Metall auf Metall. Eine andere Lösung wäre eine Art de minimis-Kriterium gewesen, vgl. BVerfG, Urt. v. 31.05.2016 - 1 BvR 1585/13, Rn. 110 - Metall auf Metall; Leistner JZ 2014, 846, 849; ders. GRUR 2019, 1008, 1009.

27 S. Leistner GRUR 2019, 1008, 1010. 
Dieser folgt auch der BGH und stellt für die Frage der Wiedererkennbarkeit auf das Hörverständnis eines durchschnittlichen Musikhörers ab. ${ }^{28}$ Im Nachgang des Urteils die Wiedererkennbarkeit im Lichte der Grundrechte zu einem von Investitionsschutzgedanken getragenen Wesentlichkeitskriterium zu dehnen, hätte dem Bestreben des EuGH widersprochen. ${ }^{29}$ Überzeugend entnimmt Leistner dem EuGH-Urteil, dass die Darlegungs- und Beweislast für die Wiedererkennbarkeit beim Anspruchssteller liegt, da diese eine immanente schutzrechtsbezogene Begrenzung des Verwertungsrechts ist. Wichtig ist, dass hier aufgrund der vollständigen Harmonisierung des materiellen Gehalts des Tonträgerherstellerrechts aus Art. 2 lit. c RL 2001/29 den Mitgliedstaaten kein Umsetzungsspielraum verbleibt. Das heißt auch, dass das Recht zur Vervielfältigung des $\$ 85$ I 1 Fall 1 UrhG richtlinienkonform auszulegen und alleine an den Grundrechten der EU-Grundrechtecharta, nicht an deutschen Grundrechten, zu messen ist. ${ }^{30}$ Damit bleibt es bei einem objektiven und recht strengen Verständnis der Vervielfältigung, auch bei Übernahme von kleinen und veränderten, wiedererkennbaren Teilen eines urheberrechtlich geschützten Werks.

\subsubsection{Verbreitung}

Eine andere Frage ist, ob der Tonträger, in den das Fragment übernommen wird, mit einer „Kopie“ i.S.v. Art. 9 I lit. b RL 2006/115 gleichzusetzen ist. Dabei geht es darum, ob neben dem Eingriff in das Vervielfältigungsrecht auch in das Verbreitungsrecht, also in ein weiteres Verwertungsrecht des Tonträgerherstellers, eingegriffen wird. Die Übernahme eines Fragments, das von einem Tonträger übernommen wurde, führt nach dem EuGH nicht zu einer „Kopie“ des Tonträgers, da weder der gesamte Tonträger noch ein wesentlicher Teil davon übernommen werden. ${ }^{31} \mathrm{Im}$

28 BGH, Urt. v. 30.04.2020 - I ZR 115/16, Rn. 25, 29 - Metall auf Metall IV m.w.N.; vgl. auch Apel MMR 2019, 601, 602; Leistner GRUR 2019, 1008, 1010; Wagner MMR 2019, 727, 729.

29 Überzeugend Leistner GRUR 2019, 1008, 1010.

30 BGH, Urt. v. 30.04.2020 - I ZR 115/16, Rn. 23 f. - Metall auf Metall IV.

31 EuGH, Urt. v. 29.07.2019 - C-476/17, NJW 2019, 2913 Rn. 54 f. - Metall auf Metall; Leistner GRUR 2019, 1008, 1011; Schulze NJW 2019, 2918 scheint demgegenüber die Wesentlichkeit beim nachschaffenden Produkt zu prüfen. Wir verstehen EuGH und BGH hingegen so, dass sie - zurecht - die Wesentlichkeit auf das ursprüngliche Produkt beziehen. 
Zuge von Appropriation Art mit Tonträgern könnte die Verbreitung jedoch zusätzlich relevant werden, solange die Übernahme die Hürde eines wesentlichen Teiles überschreitet.

Der BGH schloss sich dem Ergebnis des EuGH an. ${ }^{32}$ Der BGH stellt ferner klar, dass auch $\$ 96$ I UrhG in diesem Fall nicht "durch die Hintertür“ zu einem Verbot der Verbreitung führen kann. \$96 I UrhG, wonach rechtswidrig hergestellte Vervielfältigungsstücke nicht verbreitet werden dürften, ist richtlinienwidrig und daher unanwendbar, soweit er trotz Nicht-Verletzung des Verbreitungsrechts zu einem Verbot der Handlungsmodalität der Verbreitung führen würde. ${ }^{33}$

\subsubsection{Abschließende Wirkung der Schranken des Art. 5 RL 2001/29}

Weiterhin verdeutlicht der EuGH erneut, dass die Ausnahmen und Beschränkungen des Art. 5 RL 2001/29 abschließend sind. ${ }^{34}$ Das heißt, für Ausnahmen und Beschränkungen - nach deutschem urheberrechtlichen Verständnis Schranken - neben den im Wortlaut des Art. 5 angelegten, besteht kein Raum. Der EuGH erwähnt hier auch $\$ 24 \mathrm{I}_{\text {UrhG }}^{35}$, äußert jedoch nicht explizit, dass es sich dabei um eine nicht unionsrechtskonforme Ausweitung der Beschränkungen und Ausnahmen ${ }^{36}$ oder um eine nicht in Art. 5 RL 2001/29 angelegte Ausnahme oder Beschränkung handelt. Der BGH entschied in der Folge mit einem Paukenschlag, dass $\$ 24$ I UrhG auf klassische Fallkonstellationen der freien Benutzung nicht (mehr) anwendbar ist. ${ }^{37}$ Damit verbleibt nur eine Anwendung des $\$ 24$ I UrhG, soweit er

32 BGH, Urt. v. 30.04.2020 - I ZR 115/16, Rn. 77 - Metall auf Metall IV.

33 BGH, Urt. v. 30.04.2020 - I ZR 115/16, Rn. 80 - Metall auf Metall IV; Grünberger in Schricker/Loewenheim $\$ 96$ Rn. 9; a.A. Schulze NJW 2019, 2918; offen J.B. Nordemann in Fromm/Nordemann $\$ 96$ Rn. 3.

34 EuGH, Urt. v. 16.11.2016 - C-301/15, EuZW 2017, 238 Rn. 34 - Soulier und Doke; EuGH, Urt. v. 07.08.2018 - C-161/17, NJW 2018, 3501 Rn. 16 - Renckhoff; EuGH, Urt. v. 29.07.2019 - C-476/17, NJW 2019, 2913 Rn. 58 - Metall auf Metall.

35 EuGH, Urt. v. 29.07.2019 - C-476/17, NJW 2019, 2913 Rn. 56 - Metall auf Metall.

36 Dazu EuGH, Urt. v. 10.04.2014 - C-435/12, GRUR 2014, 546 Rn. 27 - ACI Adam u.a.; EuGH, Urt. v. 29.07.2019 - C-476/17, NJW 2019, 2913 Rn. 64 - Metall auf Metall.

37 BGH, Urt. v. 30.04.2020 - I ZR 115/16, Rn. 32, 36 - Metall auf Metall IV, im Fall stand wegen der Benutzung eines Tonträgers eine entsprechende Anwendung im Raum, Rn. 35; zur entsprechenden Anwendung von $\$ 24$ UrhG auf verwandte Schutzrechte und krit. im Fall von $₫ 85$ UrhG A. Nordemann in Fromm/Nordemann $\$ 24$ Rn. $4 \mathrm{bf}$. 
die Ausnahmen und Beschränkungen zu Parodien und Karikaturen, Art. 5 III lit. k RL 2001/29, umsetzt. $^{38}$

\subsubsection{Zitatfreibeit}

Neben der zunehmenden Verlagerung der Diskussion hin zur Auslegung der Verwertungsrechte ist für Appropriation Art entscheidend, wie bereits die Germania 3-Rechtsprechung aufgezeigt hat, ob Ausnahmen oder Beschränkungen (bzw. Schranken) eingreifen, also die Frage, ob die Nutzung doch erlaubt ist. Die Zitatfreiheit zählt zu den Ausnahmen und Beschränkungen, die in Art. 5 RL 2001/29 angelegt sind.

\subsubsection{Die Zitatfreiheit in EuGH Metall auf Metall und BGH Metall auf Metall IV}

Der EuGH definiert das Zitat als Nutzung eines Werkes oder ganz allgemein eines Auszugs aus einem Werk von einem Nutzer, der nicht dessen Urheber ist, „um Aussagen zu erläutern, eine Meinung zu verteidigen oder eine geistige Auseinandersetzung zwischen dem Werk und den Aussagen des Nutzers zu ermöglichen, so dass der Nutzer eines geschützten Werks, der sich auf die Ausnahme für Zitate berufen will, das Ziel verfolgen muss, mit diesem Werk zu interagieren. " ${ }^{39}$ Im Vordergrund steht also die Zweckbestimmung („um“) und die geistige Interaktion mit dem ursprünglichen Werk. Interessanterweise kommt dieser geistigen Auseinandersetzung bei Appropriation für das Bundesverfassungsgericht im Germania 3-Beschluss nicht derselbe Stellenwert zu. ${ }^{40}$ Ein Zitat sei aber ausgeschlossen in Situationen, in denen das potenziell zitierte Werk nicht erkennbar sei - dann könne es die erforderliche Interaktion nicht geben. ${ }^{41}$

Darüber hinaus muss das Zitat die weiteren Voraussetzungen des Art. 5 III lit. d RL 2001/29 erfüllen. Dafür muss die Nutzung insbesondere „den anständigen Gepflogenheiten“ entsprechen und ,in ihrem Umfang durch den besonderen Zweck gerechtfertigt“ sein. Ebenso muss ausweislich des

38 BGH, Urt. v. 30.04.2020 - I ZR 115/16, Rn. 38, 61 f. - Metall auf Metall IV.

39 EuGH, Urt. v. 29.07.2019 - C-476/17, NJW 2019, 2913 Rn. 71 - Metall auf Metall.

40 S.o. Ziff. 4.2; BVerfG, Beschl. v. 29.06.2000 - 1 BVR 825/98, Rn. 27 - Germania 3.

41 EuGH, Urt. v. 29.07.2019 - C-476/17, NJW 2019, 2913 Rn. 73 f. - Metall auf Metall; ebenso BGH, Urt. v. 30.04.2020 - I ZR 115/16, Rn. 54 - Metall auf Metall IV. 
Wortlauts der Schutzgegenstand bereits rechtmäßig öffentlich zugänglich gemacht worden sein und, außer in Fällen, in denen sich dies als unmöglich erweist, die Quelle, einschließlich des Namens des Urhebers, angegeben werden.

Die Prüfung des BGH orientiert sich stark an der des EuGH. Auch für den BGH steht der Zweck der geistigen Auseinandersetzung und Interaktion mit dem zitierten Werk im Vordergrund seiner Prüfung von $₫ 57$ S. 1 und 2 Nr. 3 UrhG. Der BGH konstatiert in Metall auf Metall IV, dass das übernommene Audiofragment zwar wiedererkennbar, aber nicht als fremd erkennbar ist und lehnt deshalb mangels der Interaktion mit dem genutzten Werk ein Eingreifen des Zitatrechts ab. ${ }^{42}$

\subsubsection{Einordnung der Rechtsprechung zur Zitatfreiheit}

Es spricht viel dagegen, dass die Zitatfreiheit zur Rechtfertigung der Übernahme des etwa 2-sekündigen Samples in Metall auf Metall heranzuziehen ist. Durch das schlichte Abspielen des Samples im Loop ist die erforderliche Zweckbestimmung für ein Zitat, die Interaktion mit dem ursprünglichen Schutzgegenstand, im vorliegenden Fall zweifelhaft. Ebenso scheint die Voraussetzung der Angabe der Quelle nicht erfüllt - „Metall auf Metall“ hätte auf dem Tonträger, auf dem „Nur mir“ veröffentlicht wurde, problemlos erwähnt werden können. ${ }^{43}$

Auch wenn der EuGH an dieser Stelle bei Sampling und Tonträgerherstellerrecht dazu zu tendieren scheint, die Rechtmäßigkeit vom Eingreifen des Zitatrechts abhängig zu machen, zeigt der Blick auf Appropriation Art im Allgemeinen: Das Zitatrecht allein vermag keine umfassende Antwort zu geben, sondern wirkt wie eine künstliche Verdichtung. So bezieht sich die "Lösung“ auf Basis von EuGH Metall auf Metall bisher auf die Übernahme von Fragmenten, die (1) erkennbar oder nicht erkennbar sind und deshalb in das Vervielfältigungsrecht des Tonträgerherstellers eingreifen oder nicht und (2) durch das Zitatrecht gerechtfertigt sind oder nicht. Dies spiegelt auch die Prüfung des BGH wider, wonach das Audiofragment nicht als fremd erkennbar gewesen sei und es daher an der geistigen Interaktion fehle. ${ }^{44}$ Im Falle des Samplings kann dies als ausgewogene Lösung

42 BGH, Urt. v. 30.04.2020 - I ZR 115/16, Rn. 53 ff. - Metall auf Metall IV.

43 Ebenso zur mangelnden geistigen Auseinandersetzung und Quellenangabe: Schulze NJW 2019, 2918.

44 BGH, Urt. v. 30.04.2020 - I ZR 115/16, Rn. 53 ff. - Metall auf Metall IV. 
betrachtet werden ${ }^{45}$ während Appropriation Art insgesamt nicht nur auf dem Bein des Zitatrechts stehen kann. ${ }^{46}$ Zwar hat das Zitatrecht im künstlerischen Bereich einen weiteren Anwendungsbereich als bei nichtkünstlerischen (Sprach-)werken. ${ }^{47}$

Doch: $\mathbb{} 24$ I UrhG hätte aus unserer Sicht eine ausgewogene Grundlage und hinreichende Rechtssicherheit durch über Jahrzehnte gefestigte Rechtsprechung geboten. \$24 I UrhG ist die gesetzgewordene Form eines der wesentlichsten Grundgedanken in unserem Kulturschaffen, nämlich, dass kulturelles Schaffen nicht ohne ein Aufbauen auf früheren Leistungen anderer Urheber denkbar ist. ${ }^{48}$ Regelungen wie $\$ 24$ UrhG finden sich im UrhG in ähnlicher oder abgewandelter Form seit dem preußischen Gesetz von 1837.49 Vor allem aber - und das spielt auch bei der EU-rechtlichen Bewertung eine Rolle - stellt $\$ 24$ UrhG anders als $\$ 51$ UrhG nach unserer Auffassung gerade keine Begrenzung des Schutzbereichs des Urheberrechts dar, keine urheberrechtliche Schranke, sondern definiert den Schutzbereich des Urheberrechts. ${ }^{50}$ Er ist - um in den berühmten Beispiel Ulmers vom urheberrechtlichen Baum zu bleiben - ein Ast oder ein Teil des Stammes, der gerade nicht in die beiden Äste der Persönlichkeitsrechte und Verwertungsrechte, die ggf. Schranken unterliegen, ausgreift. Damit unterfällt $\mathbb{2} 24$ UrhG auch nicht dem unionsweit harmonisierten Teil des Urheberrechts. ${ }^{51}$ Auch RBÜ und TRIPS überlassen Abgrenzung von Bearbeitung zu freier Benutzung dem Recht des Schutzlandes über Art. 5 II S. 2 RBÜ.52 Dieses über Jahrzehnte entwickelte urheberrechtliche Institut scheint uns für Fälle wie Appropriation Art weit besser geeignet als das auf spezifische Zwecke gerichtete Zitatrecht.

Im umgekehrten Fall ist jedoch zumindest klar: Wann immer das Zitatrecht eingreift und die Appropriation rechtfertigt ${ }^{53}$, bedarf es hierfür keiner weiteren Grundlage.

45 So Leistner GRUR 2019, 1008, $1013 \mathrm{f}$.

46 Vgl. dazu Döhl UFITA 2019, 19, 26, der das „Verstecken“ des Microsamples und die Rechtfertigung wegen des Zitatrechts als „Ausnahmen von geringem praktischen Anwendungsbereich" bezeichnet.

47 BVerfG, Beschl. v. 29.06.2000 - 1 BVR 825/98, Rn. 22 - Germania 3; BGH, Urt. v. 30.11.2011 - I ZR 212/10, juris Rn. 14 - Blühende Landschaften.

48 Loewenheim in Schricker/Löwenheim $\$ 24$ Rn. 2; so auch BGH, Urt. v. 30.04.2020 - I ZR 115/16, Rn. 34 - Metall auf Metall IV.

49 Chakraborty, Das Rechtsinstitut der freien Benutzung im Urheberrecht, 15 ff.

50 Vgl. Chakraborty, a.a.O, 29.

51 A. Nordemann in Fromm/Nordemann $\$ 24$ Rn. 6.

52 W. Nordemann/Vinck/Hertin Art. 12 RBÜ Rn. 1.

53 Dazu auch Dustmann in Fromm/Nordemann $\$ 51$ Rn. 41. 


\subsubsection{Die freie Benutzung nach $\$ 24$ I UrhG}

Während Bearbeitung und Umgestaltung nach der Rechtsprechung des BGH besondere Fälle der „Verarbeitung“ ein und desselben Werkes sind, führt die in freier Benutzung eines Werkes geschaffene Gestaltung nach \$24 I UrhG zu einem selbstständigen neuen Werk. ${ }^{54}$ Dieses neue Werk verletzt dann nicht die Rechte der Rechtsinhaber der Vorlage.

\subsubsection{Prüfungsmaßstab von $₫ 24$ I UrhG}

Die freie Benutzung setzt einen Abstand des neuen Werks zu den entlehnten eigenpersönlichen Zügen des benutzten Werks voraus. Gerade die entlehnten eigenpersönlichen Züge müssen verblassen. ${ }^{55}$ Ein Verblassen kann durch das Zurücktreten der eigenpersönlichen Züge im Gesamteindruck oder einen inneren Abstand zum älteren Werk eintreten. Dann handelt es sich um ein selbstständiges Werk, anderenfalls um eine bloße Vervielfältigung oder eine unfreie Bearbeitung i.S.v. $\$ 23$ UrhG. Bei einer möglichst identischen, nicht als solcher erkennbaren Kopie ohne Abstand bewegt sich die Künstler:in im Extremfall, wenn sie die Übernahme nicht angibt und unter dem Namen des Urhebers des Ausgangswerkes agiert, im Bereich von Plagiat und Fälschung, anstatt auf eine erlaubte Nutzung nach $\$ 24$ I UrhG hoffen zu können. ${ }^{56}$ Dieser Maßstab war prädestiniert für eine flexible, interessengerechte Anwendung auf Appropriation Art.

Nennenswert ist außerdem, dass $\mathbb{2} 24$ I UrhG für Parodien, Karikaturen und Pastiches nicht voraussetzt, dass die neue Gestaltung selber Werkcharakter hat. ${ }^{57}$ Im Zuge der Parodie ist nach BGH und EuGH stattdessen ne-

54 BGH, Urt. v. 28.07.2016 - I ZR 9/15, juris Rn. 17 - auf fett getrimmt.

55 St. Rspr. BGH, Urt. v. 28.07.2016 - I ZR 9/15, juris Rn. 18 m.w.N. - auf fett getrimmt.

56 Dazu und mit Beispielen, die in diese Richtung tendieren Schack FS Nordemann, $107 \mathrm{ff}$.

57 BGH, Urt. v. 28.07.2016 - I ZR 9/15, juris Rn. 28 - auf fett getrimmt (nach Ansicht von BGH, Urt. v. 30.04.2020 - I ZR 115/16, Rn. 65 - Metall auf Metall IV wurden Pastiches jedoch nie unter $₫ 24$ I UrhG gefasst); im Anschluss an EuGH, Urt. v. 03.09.2014 - C-201/13, juris Rn. 33 - Deckmyn und Vrijheidsfonds/ Vandersteen u.a. 
ben einem Ausdruck für Humor oder Verspottung erforderlich, dass wahrnehmbare Unterschiede zur Vorlage bestehen..$^{58}$

\subsubsection{Ist $\$ 24$ I UrhG unionsrechtswidrig?}

Nach EuGH Metall auf Metall stellte sich die Frage: Kann man sich überhaupt weiterhin auf $₫ 24$ UrhG beziehen? Ist $₫ 24$ UrhG für Appropriation Art überhaupt noch „nutzbar"?

Einerseits könnte $\mathbb{2 4}$ I UrhG als Ausweitung der Ausnahmen und Beschränkungen des Urheberrechts über Art. 5 I RL 2001/29 hinaus angesehen werden. Dann wäre $\$ 24$ I UrhG unionsrechtswidrig. ${ }^{59}$ Im Einklang mit dieser Ansicht bedürfte es konsequenterweise einer Neuregelung der Schranken im deutschen Recht, die $\$ 24$ I UrhG in seiner jetzigen Gestalt entfallen lässt und auch als Reaktion auf Art. 17 VII Ua. 2 lit. a, b DSMRL europarechtskonforme Schranken einführt. ${ }^{60}$

Andererseits könnte $\mathbb{2 4}$ I als allgemeine Grundrechtsschranke außerhalb der in RL 2001/29 geregelten Schranken eingestuft werden. ${ }^{61}$ Nach dieser Ansicht grenzt $\$ 24$ I UrhG den Schutzumfang gegenüber einer unfreien Bearbeitung in einem bisher nicht harmonisierten Bereich ${ }^{62} \mathrm{ab}^{63}$ Danach könnte $\$ 24$ I UrhG in einem nicht harmonisierten Bereich die Benutzung erlauben. Das fremde Werk müsse nur als Anregung genutzt wer-

58 BGH, Urt. v. 28.07.2016 - I ZR 9/15, juris Rn. 29f. - auf fett getrimmt; EuGH, Urt. v. 03.09.2014 - C-201/13, juris Rn. 33 - Deckmyn und Vrijheidsfonds/ Vandersteen u.a.

59 So Leistner GRUR 2019, 1008, 1011, 1014 f.; einschränkend Dreier GRUR 2019, 1003,1005 , nur, soweit es sich bei $\$ 24$ UrhG nicht lediglich um eine Schutzrechtsbegrenzung handele.

60 Dazu Leistner GRUR 2019, 1008, 1015; Herauslösung und Neuregelung von Parodie und Pastiche: Dreier GRUR 2019, 1003, 1005; BMJV, Entwurf eines Zweiten Gesetzes zur Anpassung des Urheberrechts an die Erfordernisse des digitalen Binnenmarkts, Diskussionsentwurf Stand 24.06.2020, S. 55 f., 64 f., https://www.bmjv .de/SharedDocs/Gesetzgebungsverfahren/Dokumente/DiskE_II_Anpassung\%20U rheberrecht_digitaler_Binnenmarkt.pdf?_blob=publicationFile\&v=2 (zuletzt abgerufen am 17.07.2020).

61 Vgl. Schulze NJW 2019, 2918; ders. in Dreier/Schulze \$24 Rn. 1; ders. FS Walter, 504, $507 \mathrm{ff}$.

62 Zur nur partiellen Harmonisierung des Bearbeitungsrechts: Schulze in Dreier/ Schulze $\$ 24$ Rn. 1a; Zweifel äußernd angesichts der Vorlage zu Metall auf Metall: A. Nordemann in Fromm/Nordemann $\$ 24$ Rn. 6a.

63 Schulze NJW 2019, 2918. 
den und hinter dem neuen Werk zurückbleiben. ${ }^{64}$ Diese Ansicht bietet insofern eine Angriffsfläche, als sie mittelbar doch so verstanden werden könnte, zu einer über Art. 5 I RL 2001/29 hinausgehenden und damit gegen Unionsrecht verstoßenden Ausnahme oder Beschränkung zu führen. Faktisch ist zuzugeben: Durch $\$ 24$ I UrhG können Nutzungen erlaubt sein, die von Art. 5 I RL 2001/29 nicht erfasst sind. Nach unserer oben vertretenen Auffassung, dass es sich bei $\$ 24$ UrhG aber nur um eine Definition des Schutzbereichs des Urheberrechts handelt, keine Beschränkung, schadet das nicht.

Der EuGH bejaht in Metall auf Metall eine Vervielfältigung und führt aus, dass die Mitgliedstaaten keine Ausnahme oder Beschränkung des Rechts des Tonträgerherstellers über Art. 5 RL 2001/29 hinaus vorsehen dürfen. ${ }^{65}$ Zudem nimmt der BGH mittlerweile an, dass Bearbeitung und Umgestaltung besondere Fälle der Vervielfältigung darstellen. ${ }^{66}$ Davon auszugehen, dass trotz der Vervielfältigungshandlung, ${ }^{67}$ die begrenzt wird durch in Art. 5 RL 2001/29 abgeschlossen geregelte Schranken, $\$ 24$ I UrhG die Benutzung in einem nicht harmonisierten Bereich gestattet, wäre nach EuGH Metall auf Metall zwar unter Umständen weiterhin möglich gewesen, aber dogmatisch klärungsbedürftig. Mit der von uns vertretenen Lösung einer Inhaltsregelung hätte dies allerdings gelingen können. ${ }^{68}$

Das sieht der BGH nun jedoch anders: Obwohl das Recht der freien Benutzung eine dem Urheberrecht immanente Beschränkung des Schutzbereichs bezeichne, sei es nicht mehr zulässig, anzunehmen, der Schutzbereich eines der Verwertungsrechte aus Art. 2 bis 4 RL 2001/29 werde durch $\$ 24$ I UrhG in der Weise (immanent) beschränkt, dass ein selbstständiges Werk, das in freier Benutzung des Werks oder der Leistung eines Rechteinhabers geschaffen worden ist, ohne seine Zustimmung verwertet werden darf. ${ }^{69}$ Das heißt im Klartext: $\mathbb{2} 24$ I UrhG kann nach der Rechtsprechung nicht mehr allgemein die freie Benutzung von Werken erlauben - eine allgemeine freie Benutzung hält der BGH für unionsrechtswidrig.

64 Schulze in Dreier/Schulze $\$ 24 \mathrm{Rn} .1 \mathrm{a}$.

65 EuGH, Urt. v. 29.07.2019 - C-476/17, NJW 2019, 2913 Rn. 65 - Metall auf Metall.

66 BGH, Urt. v. 28.07.2016 - I ZR 9/15, juris Rn. 17 - auf fett getrimmt; ähnlich bereits BGH, Urt. v. 16.05.2013 - I ZR 28/12, GRUR 2014, 65, 70 Rn. 36 - BeuysAktion; kritisch A. Nordemann in Fromm/Nordemann $\$ 24$ Rn. 9 f.

67 Die Harmonisierung des Vervielfältigungsbegriffs könnte sich auf den Schutzumfang des Urheberrechts auswirken, Jan Bernd Nordemann FS Mathias Schwarz, 97, $99 \mathrm{ff}$.

68 Vgl. EuGH, Urt. v. 29.07.2019 - C-476/17, NJW 2019, 2913 Rn. 56 ff. - Metall auf Metall.

69 BGH, Urt. v. 30.04.2020 - I ZR 115/16, Rn. 36 - Metall auf Metall IV. 
Diese Rechtsprechung ist nicht als auf das Tonträgerherstellerrecht begrenzt zu verstehen. Die abschließende Regelung der Ausnahmen und Beschränkungen in Art. 5 RL 2001/29 ist das tragende Argument für die Unionsrechtswidrigkeit des dort nicht angelegten $\$ 24$ I UrhG. $^{70}$ Dieser abschließende Charakter gilt nicht nur für das Tonträgerherstellerrecht, sondern allgemein für die Verwertungsrechte der Rechtsinhaber aus Art. 2 bis 4 RL 2001/29.71

\subsubsection{Die unionsrechtskonforme Auslegung von $\$ 24$ I UrhG}

Nur insofern, als $\$ 24$ I UrhG eine der in Art. 5 RL 2001/29 abschließend geregelten Ausnahmen oder Beschränkungen umsetzt, verbleibt nach der neuen Rechtsprechung des BGH ein Anwendungsbereich für $\$ 24$ I UrhG. Dieser Restanwendungsbereich beschränkt sich auf Karikaturen und Parodien. ${ }^{72}$ Schon vorher hatte der BGH trotz der EuGH-Rechtsprechung zum Satirebegriff ${ }^{3}$ keinen Anlass gesehen, entsprechende Fallgestaltungen nicht mehr über $\$ 24$ I UrhG zu lösen. Vielmehr ging der BGH den Weg einer richtlinienkonformen Auslegung des $\$ 24$ I UrhG. ${ }^{74}$

Dabei erwähnte der BGH im Zusammenhang mit der unionsrechtskonformen Auslegung des $\$ 24$ I UrhG bereits ausdrücklich Pastiches. ${ }^{75}$ Einer Anwendung von $\$ 24$ I UrhG als Pastiche-Schranke schob der BGH jetzt gleichsam einen Riegel vor. Es sei grundsätzlich allein eine Sache des Gesetzgebers und nicht der Gerichte, eine nach Art. 5 III lit.k RL 2001/29 mögliche Pastiche-Schranke in innerstaatliches Recht umzusetzen. ${ }^{76}$

70 BGH, Urt. v. 30.04.2020 - I ZR 115/16, Rn. 36 - Metall auf Metall IV.

71 Vgl. EuGH, Urt. v. 16.11.2016 - C-301/15, EuZW 2017, 238 Rn. 34 - Soulier und Doke; EuGH, Urt. v. 07.08.2018 - C-161/17, NJW 2018, 3501 Rn. 16 - Renckhoff; BGH, Urt. v. 30.04.2020 - I ZR 115/16, Rn. 36 - Metall auf Metall IV, „Rechtsinhaber“; ErwGr. 32 RL 2001/29.

72 Vgl. BGH, Urt. v. 30.04.2020 - I ZR 115/16, Rn. 32, 61 f. - Metall auf Metall IV.

73 EuGH, Urt. v. 03.09.2014 - C-201/13 - Deckmyn und Vrijheidsfonds/Vandersteen u.a.

74 BGH, Urt. v. 28.07.2016 - I ZR 9/15, juris Rn. 24 - auf fett getrimmt; ebenso: Unseld EuZW 2014, 912, 915; Lauber-Rönsberg ZUM 2015, 658, 665; Haedicke GRUR Int. 2015, 664, 670; der Sache nach ebenso Slopek GRUR-Prax 2014, 442; von Becker GRUR 2015, 336, 339; vgl. auch Peifer jurisPR-WettbR 2015 Anm. 1 unter D.

75 BGH, Urt. v. 28.07.2016 - I ZR 9/15, juris Rn. 28 - auf fett getrimmt.

76 BGH, Urt. v. 30.04.2020 - I ZR 115/16, Rn. 64 f. - Metall auf Metall IV. 
Was genau ist aber ein Pastiche? Eine Beschäftigung damit ist nach dem Urteil des BGH weiter angezeigt, denn: Gerade aufgrund der Lücke, die die Aushöhlung von $\$ 24$ I UrhG hinterlässt, bedarf es gesetzgeberischer Neuregelungen, die künstlerisches Schaffen auffangen, welches nach der neuen Rechtsprechung ansonsten nicht mehr gestattet wäre. So schlägt bereits der neue Diskussionsentwurf des BMJV zur Umsetzung der DSMRichtlinie die Schaffung einer Pastiche-Schranke in $\$ 51 \mathrm{a}$ UrhG-E vor. ${ }^{77}$ Obwohl der Begriff des Pastiches sich in den Ausnahmen und Beschränkungen in Art. 5 III lit. k der RL 2001/29 wiederfindet, ist der (unionsrechtliche) Bedeutungsgehalt alles andere als klar: weder nationenübergreifend noch kunstwissenschaftlich bzw. -historisch oder über verschiedene Kunstformen hinweg. ${ }^{78}$

Der Generalanwalt Szpunar schreibt in den Schlussanträgen zum Verfahren Metall aufMetall: "Was den Begriff des Pastiches betrifft, bezeichnet dieser eine Nachahmung des Stils eines Werkes oder eines Urhebers, ohne dass notwendigerweise Bestandteile dieses Werks übernommen werden." 79 Würde diese Einschätzung als Definition herangezogen, ließe sich durch die Nachahmung des Stils eines Werkes oder Urhebers bei optionaler Übernahme von Werkbestandteilen ein nicht zu unterschätzender Bereich von Appropriation darunter fassen. Eine Schwäche dieses Verständnisses ist, dass der Stil grundsätzlich nicht urheberrechtlich geschützt ${ }^{80}$ ist. $S z p u$ nar wollte den Begriff des Pastiches allerdings wohl selber nicht ausufernd verstanden wissen, indem er im Fall von Metall auf Metall kein Pastiche für möglich hielt. Aufgrund der Übernahme eines Tonträgers, um ein Werk in anderem Stil zu schaffen, verneinte er ein Pastiche. ${ }^{81}$

$77 B M J V$, Entwurf eines Zweiten Gesetzes zur Anpassung des Urheberrechts an die Erfordernisse des digitalen Binnenmarkts, Diskussionsentwurf Stand 24.06.2020, S. 10, https://www.bmjv.de/SharedDocs/Gesetzgebungsverfahren/Dokumente/Dis kE_II_Anpassung\%20Urheberrecht_digitaler_Binnenmarkt.pdf?_blob=publicati onFile\&v=2 (zuletzt abgerufen am 17.07.2020).

78 Vgl. Döhl UFITA 2019, 19, 26 ff., insb. 30 ff. m.w.N.

79 Szpunar, Schlussanträge des Generalanwalts - C-476/17, Fn. 30 - Pelham u.a./ Hütter u.a.

80 Dazu BMJV, Entwurf eines Zweiten Gesetzes zur Anpassung des Urheberrechts an die Erfordernisse des digitalen Binnenmarkts, Diskussionsentwurf Stand 24.06.2020, S. 64, https://www.bmjv.de/SharedDocs/Gesetzgebungsverfahren/Dok umente/DiskE_II_Anpassung\%20Urheberrecht_digitaler_Binnenmarkt.pdf?_blo $\mathrm{b}=$ publicationFile $\& \mathrm{v}=2$ (zuletzt abgerufen am 17.07.2020).

81 Szpunar, Schlussanträge des Generalanwalts - C-476/17, Fn. 30 - Pelham u.a./ Hütter u.a. 
Eine durchaus weite Fassung klingt im Diskussionsentwurf des BMJV an, wonach Pastiches auch Ausdruck von „Wertschätzung oder Ehrerbietung für das Original enthalten" können und insbesondere an Praktiken wie Remixes, Memes, GIFs, Mashups, Fan Art, Fan Fiction, Covers und Samplings zu denken sei. ${ }^{82}$

Ohne eine Klärung des Begriffs durch den EuGH wäre es wegen der Vielzahl an Definitionsmöglichkeiten kein verlässlicher Weg gewesen, Appropriation Art unter einen sehr weit verstandenen Pastiche-Begriff zu fassen, der in unionsrechtskonformer Auslegung des $\$ 24$ I UrhG angewendet worden wäre. Gleichwohl besteht nach dem BGH-Urteil nun gesetzlicher Handlungsbedarf in Bezug auf Pastiches. Art. 17 VII Ua. 2 lit. b DSMRL erfordert in dessen Anwendungsbereich - Diensteanbieter für das Teilen von Online-Inhalten (DTOs), z.B. YouTube - zwingend, dass die Nutzer sich auf die Nutzung zum Zwecke von Pastiches berufen können. Weil der BGH den Weg über $\$ 24$ I UrhG versperrt hat, bleibt hierfür, eine unmittelbare Anwendbarkeit des Unionsrechts außen vor gelassen, nur der Weg über eine gesetzliche Neuregelung bei der Umsetzung der DSMRL. Obwohl eine solche Pastiche-Schranke auf die Haftung von DTOs begrenzt werden könnte, wäre eine Lösung für das gesamte Urheberrecht überzeugender.

Angesichts der neuen EuGH- und BGH-Rechtsprechung bleibt in der Praxis für eine allgemeine Regelung zur freien Benutzung weder eine unionsrechtskonforme Auslegung des $\mathbb{2 4}$ I UrhG noch das Dach eines nicht harmonisierten Bereichs. Den Vorschlag, $\mathbb{2} 24$ UrhG aufzuheben und gleichzeitig $\mathbb{} \$ 23$ I UrhG-E so zu verändern, dass dieser vorherige Prüfungsmaßstäbe von $\$ 24$ I UrhG enthält (Abstand zum verwendeten Werk, Eigenart des neuen Werkes, Verblassen der entlehnten eigenpersönlichen Züge des alten Werkes), ${ }^{83}$ halten wir für nicht zielführend. Eine Regelung solchen Inhalts widerspräche nach der aktuellen Rechtsprechung genauso dem abschließenden Charakter von Art. 5 RL 2001/29 wie $\$ 24$ I UrhG.

$82 B M J V$, Entwurf eines Zweiten Gesetzes zur Anpassung des Urheberrechts an die Erfordernisse des digitalen Binnenmarkts, Diskussionsentwurf Stand 24.06.2020, S. 64, https://www.bmjv.de/SharedDocs/Gesetzgebungsverfahren/Dokumente/Dis kE_II_Anpassung\%20Urheberrecht_digitaler_Binnenmarkt.pdf?_blob=publicati onFile\&v=2 (zuletzt abgerufen am 17.07.2020).

$83 B M J V$, Entwurf eines Zweiten Gesetzes zur Anpassung des Urheberrechts an die Erfordernisse des digitalen Binnenmarkts, Diskussionsentwurf Stand 24.06.2020, S. 7, 55, https://www.bmjv.de/SharedDocs/Gesetzgebungsverfahren/Dokumente/ DiskE_II_Anpassung\%20Urheberrecht_digitaler_Binnenmarkt.pdf?_blob=publi cationFile\&v=2 (zuletzt abgerufen am 17.07 .2020$)$. 
Was dann jedoch fehlt, ist eine offenere und damit wandlungsfähigere Lösung als die Zitatfreiheit und andere Schrankenregelungen, um sich auf zahlreiche Kunstbereiche einzustellen. Das zeigt nicht zuletzt die Systematik des $₫ 51$ S. 2 Nr. 1-3 UrhG, in dem als Regelbeispiele wissenschaftliche Werke, Sprachwerke und Werke der Musik angeführt werden. Die Allgemeinheit hat ein hohes Interesse daran, mit einem Institut wie der freien Benutzung die Kunst weiterzuentwickeln und auf vorbestehenden Werken aufzubauen, auch und gerade wenn dies nicht im Wege eines Zitates erfolgt. ${ }^{84}$

\subsubsection{Zwischenfazit}

Es bedarf also einiger juristischer „Klimmzüge“, um den urheberrechtlichen Rahmen der Appropriation Art, gerade auch nach der neuen Rechtsprechung, darzustellen. Ist das eine gute Situation für ein Massenphänomen wie Appropriation Art?

Typische Konstellationen von Appropriation Art, aber auch generell das Aufbauen auf bisherigen Werken im Urheberrecht, können nicht mehr auf die generelle Grundlage des $\$ 24$ I UrhG gestützt werden. Konsequenterweise sieht der deutsche Gesetzgeber bei $\$ \$ 23,24$ UrhG und einer Pastiche-Schranke Handlungsbedarf. ${ }^{85}$ Auch eine unionsweite Harmonisierung des Bearbeitungsrechts kommt in Betracht. Unabhängig davon, anhand welcher deutschen Norm die Schaffung von Appropriation Art gemessen werden soll, zeigen die Germania 3-Rechtsprechung des Bundesverfassungsgerichts sowie das EuGH-Urteil Metall auf Metall deutlich: Die letztlich entscheidende Frage nach der Rechtfertigung bzw. erlaubnisfreien Nutzung erfordert eine Interessenabwägung der Grundrechte. Hierbei streiten auf beiden Seiten, im nationalen und im Unionsrecht, primär dieselben Grundrechtspositionen: Schutz des Eigentums und Kunstfreiheit. Jede Entscheidung über die Rechtmäßigkeit von Appropriation Art bildet den Ausgleich zwischen diesen Grundrechtspositionen ab. Eben dieser Ausgleich bildet den Reiz für das Urheberrecht, den gesellschaftlichen Diskurs, wirtschaftliche Überlegungen und nicht zuletzt auch die kritische

84 Chakraborty, Das Rechtsinstitut der freien Benutzung im Urheberrecht, $54 \mathrm{f}$.

$85 B M J V$, Entwurf eines Zweiten Gesetzes zur Anpassung des Urheberrechts an die Erfordernisse des digitalen Binnenmarkts, Diskussionsentwurf Stand 24.06.2020, S. 7, 10, 44, 54 ff., 62 ff., https://www.bmjv.de/SharedDocs/Gesetzgebungsverfahre n/Dokumente/DiskE_II_Anpassung\%20Urheberrecht_digitaler_Binnenmarkt.pdf

?_blob=publicationFile\& $\mathrm{v}=2$ (zuletzt abgerufen am 17.07.2020). 
Hinterfragung sowie den künstlerischen Schaffensprozess. Insofern ist Appropriation Art in gewisser Hinsicht auch ein Bildnis für das Verhältnis vom deutschen zum europäischen Urheberrecht: Manches kommt neu hinzu, anderes wird übernommen und vielleicht sogar verbessert, wieder anderes bleibt beim Alten - und am Ende steht hoffentlich eine ästhetische Lösung.

Wir haben allerdings Bedenken, ob die hier gefundene Lösung oder auch die anderen, die diskutiert werden, wirklich massentauglich sind und zu weiterer Akzeptanz des Urheberrechts im digitalen Zeitalter beitragen oder ob nicht vielmehr aufgrund zu komplexer Lösungen und immer neuer rechtlicher „Klimmzüge“ die Akzeptanz geistiger Eigentumsrechte gerade schwindet.

\section{Fazit}

Appropriation Art ist in Zeiten zunehmender Digitalisierung auch der Kunst ein Massenphänomen. Dennoch gibt es noch keine massentaugliche urheberrechtliche Lösung für dieses Phänomen.

Was bleibt, ist: Deutlich mehr Klarheit durch EuGH und BGH, gleichzeitig auch nach der BGH-Entscheidung nur eingeschränkt definitive Antworten zur Behandlung von Appropriation Art im deutschen Recht. Dennoch wird die grundsätzliche Linie auch im Lichte der verfassungsrechtlichen Entscheidungen Germania 3 und Metall auf Metall deutlich. Die entscheidenden urheberrechtlichen Weichenstellungen erfolgen auf Abwägungsebene. Dafür hätte $\$ 24$ I UrhG - entweder in unionsrechtskonformer Auslegung oder als nicht harmonisierte Inhaltsregelung - weiterhin die Chance geboten, künstlerischem Ausdruck durch Appropriation Art gerecht zu werden, auch und gerade in der digitalen Welt. Der Abgesang von $\$ 24$ I UrhG, bis auf Parodien und Karikaturen, hinterlässt ein Vakuum. Künstler:innen, die Appropriation Art betreiben und sich rechtssicher verhalten wollen, müssen sich nach der neuen Rechtsprechung zunächst auf ein Weniger an Rechten einstellen. Aus diesem Grund besteht für ein Institut, das Fälle der freien Benutzung gestattet, nun gesetzgeberischer, vor allem europarechtlicher, Handlungsbedarf. 


\section{Literatur}

Apel, Simon: Anmerkung zu: EuGH, Urteil vom 29.07.2019 - C-476/17 - Pelham u.a., MMR 9 (2019), S. $601 \mathrm{ff}$.

Von Becker, Bernhard: Die entstellende Parodie. Das EuGH-Urteil „Vrijheidsfonds/ Vandersteen“ und die Folgen für das deutsche Recht, GRUR 4 (2015), S. $336 \mathrm{ff}$.

Bundesministerium der Justiz und für Verbraucherschutz: Entwurf eines Zweiten Gesetzes zur Anpassung des Urheberrechts an die Erfordernisse des Digitalen Binnenmarktes, Diskussionsentwurf Stand 24. Juni 2020, https:/www.bmjv.de/Sha redDocs/Gesetzgebungsverfahren/Dokumente/DiskE_II_Anpassung\%20Urhebe rrecht_digitaler_Binnenmarkt.pdf?_blob=publicationFile\&v=2 (zuletzt abgerufen am 17.07.2020).

Bundesverband Musikindustrie: Musikindustrie in Zahlen 2018, http://www.miz.org/ downloads/dokumente/950/BVMI-2018-jahrbuch-musikindustrie-in-zahlen.pdf (zuletzt abgerufen am 17.07.2020).

Burkholder, J. Peter: Musical Borrowing or Curious Coincidence? Testing the Evidence, The Journal of Musicology 35/2 (2018), S. $223 \mathrm{ff}$., doi: 10.1525/ jm.2018.35.2.223.

Burkholder, J. Peter, Musical Borrowing \& Reworking. An Annotated Bibliography, http:/www.chmtl.indiana.edu/borrowing/ (zuletzt abgerufen am 17.07.2020).

Chakraborty, Martin, Das Rechtsinstitut der freien Benutzung im Urheberrecht, Baden-Baden 1997.

Döhl, Frédéric: Nach $\mathbb{2} 24$ Abs. 1 UrhG: Zum Pastichebegriff im Kontext der anstehenden Neuaufstellung der Spielregeln freier Benutzung, UFITA 1 (2019), S. 19 ff., doi: 10.5771/2568-9185-2019-1-19.

Dreier, Thomas: Grundrechte und die Schranken des Urheberrechts - Anmerkung zu EuGH „Funke Medien NRW“ und „Spiegel Online“, GRUR 10 (2019), S. $1003 \mathrm{ff}$.

Dreier, Thomas / Schulze, Gernot, Urheberrechtsgesetz: Verwertungsgesellschaftengesetz, Kunsturhebergesetz: Kommentar, 6. Aufl., München 2018.

Haedicke, Maximilian: Beschränkung der Parodiefreiheit durch europäisches Urheberrecht?, GRUR Int. 7-8 (2015), S. 664 ff.

Huttenlauch, Anna Blume, Appropriation Art - Kunst an den Grenzen des Urheberrechts, Baden-Baden 2010, doi: https://doi.org/10.5771/9783845224947.

Kempfert, Kamila: Referenzielles Schaffen im Lichte der Regulierung des polnischen Urheberrechtsgesetzes, UFITA 1 (2019), S. 123 ff., doi: 10.5771/25689185-2019-1-123.

Lauber-Rönsberg, Anne: Parodien urheberrechtlich geschützter Werke. Eine Bestandsaufnahme nach der »Deckmyn«-Entscheidung des EuGH, ZUM 8/9 (2015), S. $658 \mathrm{ff}$.

Leistner, Matthias: Urheberrecht in der digitalen Welt, JZ 17 (2014), S. 846 ff., doi: 10.1628/002268814X14060241209325. 
Leistner, Matthias: „Ende gut, alles gut“ ... oder „Vorhang zu und alle Fragen offen“? Das salomonische Urteil des EuGH in Sachen „Pelham [Metall auf Metall]", GRUR 10 (2019), S. $1008 \mathrm{ff}$.

Metzger, Axel: »Germania 3 Gespenster am toten Mann« oder Welchen Zweck darf ein Zitat gemäß \$51 Nr. 2 UrhG verfolgen?, ZUM 11 (2000), S. 924 ff.

Nordemann, Axel / Nordemann, Jan Bernd / Czychowski, Christian (Hrsg.), Fromm/ Nordemann, Urheberrecht, Kommentar zum Urheberrechtsgesetz, Verlagsgesetz, Einigungsvertrag (Urheberrecht), neu: zur EU-Portabilitätsverordnung, 12. Aufl., Stuttgart 2018.

Nordemann, Jan Bernd, Bearbeitung und Europarecht - Muss die Blässetheorie vor den EuGH?, in: Haesner, Christoph / Kreile, Johannes / Schulze, Gernot (Hrsg.), Zwischen Gestern und Morgen - Medien im Wandel. Festschrift für Mathias Schwarz zum 65. Geburtstag, München 2017, S. 97-110.

Nordemann, Wilhelm / Vinck, Kai / Hertin, Paul, Internationales Urheberrecht und Leistungsschutzrecht der deutschsprachigen Länder unter Berücksichtigung auch der Staaten der Europäischen Gemeinschaft: Kommentar, Düsseldorf 1977.

Peifer, Karl Nikolaus: Die neuen Freiheiten und die unsicheren Grenzen der Parodie, Anmerkung zu: EuGH Große Kammer, Urteil vom 03.09.2014 - C-201/13, jurisPR 2 (2015), Anm. 1.

Saiz-Garcia, Concepción: Ein Recht auf Referenz im spanischen Urheberrecht?, UFITA 1 (2019), S. 107 ff., doi: 10.5771/2568-9185-2019-1-107.

Schack, Haimo, Appropriation Art und Urheberrecht, in: Ulrich Loewenheim (Hrsg.), Urheberrecht im Informationszeitalter. Festschrift für Wilhelm Nordemann, München 2004, S. 107-113.

Loewenheim, Ulrich / Leistner, Matthias / Ohly, Ansgar, Urheberrecht: UrhG, KUG, VGG: Kommentar, 6. Aufl., München 2020.

Schulze, Gernot, Gedanken zur freien Benutzung und zu einer allgemeinen Grundrechtsschranke am Beispiel Metall auf Metall, in: von Lewinski, Silke / Wittmann, Heinz (Hrsg.), Urheberrecht! Festschrift für Michel M. Walter zum 80. Geburtstag, Wien 2018, S. 504-522.

Schulze, Gernot: Anmerkung zum Urteil des EuGH vom 29.7.2019 - C-476/17 (Pelham GmbH ua/Hütter ua), NJW 40 (2019), S. 2918.

Slopek, David E. F.: Begriff und Grenzen der Parodiefreiheit, GRUR-Prax 19 (2014), S. 442.

Stromberg, Matt, A Film Festival Illuminates the Experimental Edge of Appropriation Cinema, https://hyperallergic.com/531520/festival-of-inappropriation-los-ang eles-filmforum/ (zuletzt abgerufen am 17.07.2020).

Unseld, Christopher: Anmerkung zum Urteil des EuGH vom 03.09.2014 - C-201/13 (Deckmyn, Vrijheidsfonds VZW/Vandersteen ua), EuZW 23 (2014), S. $914 \mathrm{f}$.

Wagner, Kristina: Entstehung eines neuen Urheberrechtswerks am Beispiel des Musiksampling. Spannungsverhältnis mit vorbestehenden Schutzrechten, MMR 11 (2019), S. 727 ff. 
Zuschlag, Christoph, „Die Kopie ist das Original“: Über Appropriation Art, in: Mensger, Ariane (Hrsg.), Déjà-vu? Die Kunst der Wiederholung von Dürer bis YouTube. Staatliche Kunsthalle Karlsruhe, Karlsruhe 2012, S. 126-135. 